\title{
Une troisième (et dernière) révolution? Ou le tourisme comme rapport unique à l'urbain et au monde
}

A third (and last) revolution? Or tourism as the unique relationship to urbanity and the world

\section{Georges-Henry Laffont}

\section{OpenEdition \\ Journals}

Édition électronique

URL : http://journals.openedition.org/tourisme/2021

DOI : 10.4000/tourisme.2021

ISSN : 2492-7503

Éditeur

Éditions touristiques européennes

\section{Référence électronique}

Georges-Henry Laffont, « Une troisième (et dernière) révolution? Ou le tourisme comme rapport unique à l'urbain et au monde », Mondes du Tourisme [En ligne], 15 | 2019, mis en ligne le 01 juin 2019, consulté le 30 octobre 2019. URL : http://journals.openedition.org/tourisme/2021 ; DOI : 10.4000/ tourisme.2021

Ce document a été généré automatiquement le 30 octobre 2019

\section{cc)}

Mondes du tourisme est mis à disposition selon les termes de la licence Creative Commons Attribution - Pas d'Utilisation Commerciale - Pas de Modification 4.0 International. 


\title{
Une troisième (et dernière) révolution? Ou le tourisme comme rapport unique à l'urbain et au monde
}

\author{
A third (and last) revolution? Or tourism as the unique relationship to urbanity \\ and the world
}

Georges-Henry Laffont

1 Cette réflexion, dans la poursuite des travaux coordonnés par Nahrath et Stock (2012) et Duhamel (2016), ambitionne de participer à repenser la relation qu'il existe entre urbanité, tourisme et habiter. D'un côté, l'urbanité (Lussault, 2003) désigne la qualité urbaine différentielle des lieux, en appréciant le couplage de différents éléments liés aux pratiques et à leur organisation en milieu urbain (concentration, hétérogénéité, centralité, etc.). De l'autre, habiter touristiquement (Stock, 2016) serait le phénomène englobant toute présence temporaire en un lieu où se joue un certain déplacement, une distanciation de soi, des autres, de l'espace et du temps. Si le propos amène à questionner la relation qui peut s'établir entre ces deux éléments, au sens où le tourisme s'affiche en vecteur de l'urbanité contemporaine, produit de l'urbanisation planétaire du monde, mais aussi en principe actif de celles-ci (Lussault et Stock, 2007), il est ici question d'ajouter un élément fondamental, à savoir : la production de l'urbain au sens de l'établissement d'une réalité matérielle, des modalités opérationnelles de cet établissement, des idéologies et valeurs qui les sous-tendent (Lefebvre, 1974; Harvey, 2011). En effet, il paraît difficile d'explorer cette relation en faisant l'impasse sur ce qui la façonne, contraint, autorise et lui offre ses cadres d'expression.

Dans son acception la plus partagée, le tourisme implique un déplacement physique vers des lieux promettant un ailleurs. Or, cela est-il dû au fait que l'écoumène touristique se révèle plus vaste que nous pourrions le croire, au point qu' « aucun lieu n'échappe au tourisme» (Duhamel, 2016, n.p.), ou que les pratiques et attentes touristiques sont redéfinies, mais le tourisme nécessite de moins en moins un 
déplacement physique. Tout lieu, ici urbain, peut prétendre offrir un ailleurs, une expérience (Tuan 2006; Schaeffer, 2015) de distanciation du quotidien... sur place. D'ailleurs, rien de bien surprenant car, si l'on s'intéresse à l'habiter touristiquement, il faut alors reconnaître que l'être humain est capable d'être là et ailleurs, de se projeter vers des lieux où il n'est pas physiquement présent (Hoyaux, 2015) tout en éprouvant la matérialité de son quotidien. Fut un temps, la plage était, paraît-il, sous les pavés. L'aventure, quant à elle, est toujours plus ou moins au coin de la rue. Aujourd'hui, en forçant le trait, il n'est pas incongru d'avancer qu'exotisme, jeu, découverte, rencontres, etc. - dimensions d'un habiter touristiquement - sont partout et ce, d'une manière plus prononcée en milieu urbain.

3 Alors, quelles expériences conjointes de l'urbanité et de l'habiter touristiquement sont proposées dans et par la ville contemporaine? Quels "ailleurs » offrent acteurs, processus et dispositifs du projet urbain ? L'invitation faite à tout un chacun à prendre possession des différentes opérations d'aménagement urbain, tels des réaménagements de places publiques ou de quais, l'inauguration d'un quartier sur une ancienne friche, la reconversion d'un bâtiment industriel en pôle de créativité, peut-elle être considérée comme une exhortation à faire du tourisme chez soi? Quelles logiques et quelles finalités président à l'essor de cette esthétisation festive de la politique urbaine?

Émergent deux hypothèses sur les relations d'interdépendance et de tensions entre urbanisation et touristification, illustrant un nouveau stade du "recreational turn " (Stock, 2007). Tout d'abord, la ville deviendrait une ville faite uniquement pour le tourisme (Laffont et Prigent, 2011). Puis, ce dernier serait le rapport privilégié et quasiexclusif au monde (Bégout, 2012). La thèse défendue ici est que tout espace urbain est apprêté par et pour le tourisme; que tout comportement urbain est recodé en appropriation et pratique touristiques et qu'enfin tout acte de projetation, urbanistique et/ou architectural, vise à produire les conditions d'un rapport au monde fondé sur le ludique, le fun.

5 S'appuyant sur une méthode exploratoire croisant la mobilisation d'un corpus théorique lié aux éléments constitutifs de nos hypothèses et thèses et une série d'observations in situ à Nantes et à Lyon, cette contribution vise aussi à porter au débat les questions sociales que soulèvent ces hypothèses et thèses ${ }^{1}$. Pour cela, nous procéderons en trois temps: nous établirons les liens dynamiques qui s'instaurent entre tourisme et ville; nous rapporterons nos premières observations faites à Nantes et à Lyon; nous discuterons des recompositions sociales induites ou réalisées à la faveur de cet apprêtage par et pour le tourisme.

\section{Tourisme et urbanisation : quels liens ? Quelles évolutions?}

6 Le tourisme, invention urbaine de la révolution industrielle (Équipe MIT, 2002), est la promesse d'un ailleurs, où chacun(e) s'installe temporairement dans une sorte de paradis insulaire. Tourisme et touriste renvoient à l'extraordinaire, l'inhabituel, au " hors-quotidien », voire à l'inversion du quotidien (Urry, 1990). L'activité touristique revient donc à créer une rupture vis-à-vis des habitudes et à installer la possibilité d'expériences nouvelles. Enfin, à la manière des vendeurs de produits cosmétiques ou 
d'ameublement d'intérieur, les acteurs touristiques promettent à leurs clients la consommation et la représentation d'une vie idéale.

7 Objet individuel et collectif de convoitise, cet ailleurs est ce qui permet de nouer un rapport intentionnel dans lequel l'individu manifeste une appétence envers un lieu: c'est l'image à laquelle «nous prêtons nos pensées, nos désirs et nos rêves" (Mondzain, 1995, p.18). Jusqu'ici, cet ailleurs regroupait un certain nombre d'archétypes, de figures, plus ou moins partagés par tous : l'exotisme, le dépaysement, le ressourcement, la découverte, le repos. Depuis plusieurs décennies, les aspirations évoluent, s'affinent, se recombinent et le modèle d'une offre touristique qualifiée «de masse ", peu segmentée et différenciée, est grandement questionnée. Une offre plus composite, plus diversifiée et plus individualisée s'institutionnalise. De même que les économies sont passées du stade du capitalisme organisé à un capitalisme diffus, le tourisme est passé de formes hautement organisées et de masse, caractéristiques de l'après-guerre, à des modalités plus éclatées (Lash et Urry, 1994). De la sorte, au sein de ce vaste éventail de produits/services d'un ailleurs, la pratique du tourisme urbain, pourtant une forme ancienne de l'activité touristique, s'est renouvelée de manière spectaculaire depuis trois décennies (Duhamel, 2016).

8 Ce renouveau a permis à de nombreuses villes d'accroître leur attractivité. Par divers réinvestissements qualifiés d'autant de "régénération", «réhabilitation", "transformation", « renouvellement", les acteurs de l'urbain, soucieux d'insuffler de l'« imaginaire au cœur de l'ordinaire» (Urbain, 1998, p. 120), visent à attribuer à des espaces des rôles de (dé)monstrateurs d'attractivité, de conformité mais aussi de distinction au regard des discours sur l'urbain contemporain (Adam et Laffont, 2018). Sont ainsi produits des clusters de créativité, des cool places ou des lieux branchés (Richards, 2011; Maitland, 2010), où art et douceur de vivre sont mis en avant bien plus que la présence d'un patrimoine architectural, urbain ou culturel reconnu. Afin de rendre le territoire attractif, la culture et le tourisme sont ainsi utilisés pour (re)valoriser des espaces en déclin et s'insèrent tous deux dans des opérations d'urbanisme de grande envergure (Gravary-Barbas, 2013). Dans cette logique et dynamique, l'éclosion de grands projets et l'organisation d'évènements, pouvant être mis en récit et images, permettent aux villes non seulement la promotion de l'espace public comme lieu par excellence d'une urbanité à la fois réactivée et rénovée, mais surtout « d'accroître leurs marques de distinction afin de mieux asseoir leur prétention à l'unicité, source de rentes de monopoles » (Harvey, 2011, p. 45).

9 Si le tourisme n'échappe donc pas aux changements induits par la mondialisation et la métropolisation, l'individuation et l'hybridation des aspirations et attentes (GravariBarbas et Fagnoni, 2013) les façonnent également. Aujourd'hui, une attention renouvelée est portée à ce qui relève de l'ordinaire des lieux et des modes d'habiter qui s'y déploient quotidiennement. Dans l'habiter touristiquement, ce qui importe est la contextualisation du séjour et notamment la potentialité d'interactions sociales, d'expériences authentiques (Andersson, 2007 ; Sallet-Lavorel, 2003). Et cette attention s'exprime surtout envers toutes les villes qui s'affirment en autant de destinations phares du tourisme contemporain. Dès lors, garantir cet ailleurs devient un nouvel enjeu, tant pour les acteurs locaux que pour ceux qui créent les destinations tendances et proposent les dispositifs correspondant aux attentes (Airbnb, CityPASS, City Tours, vols low cost, etc.). Ainsi, les relations entre villes, tourisme et touristes se traduisent dès lors par la multiplication des lieux (culturels, ludiques, de nature, etc.), la 
diversification des activités proposées (affaires, agrément, famille, sport), une plus grande variabilité des séjours (du séjour de moins de 24 heures en passant par le city break d'un week-end au séjour d'une semaine).

Toutefois, la ville festive (Gravari-Barbas, 2000), lieu de divertissement, de culture ou de loisir, se doit, au-delà d'une attractivité retrouvée et amplifiée, de redonner à ses résidents le plaisir d'être en ville. Pour relever ces deux enjeux, antagoniques, s'inventent alors de nouveaux modes de dire, de faire, de vivre et de consommer la ville. Par une sérendipité ${ }^{2}$ potentiellement plus forte mais aussi par un partage de l'espace et des ressources parfois conflictuels (dans certains quartiers parisiens, ou celui de la Croix-Rousse à Lyon), se redéfinissent ainsi tourisme, urbanité et habiter.

Dans cette perspective, on peut alors concevoir l'habiter touristiquement comme l'élément majeur du processus d'urbanisation. En effet, la mise en tourisme des villes correspond à un processus d'augmentation du degré d'urbanité, de la requalification de l'espace public ou encore de la mise en place d'une nouvelle centralité au-delà du bassin de vie. Parallèlement, d'une urbanité définie par l'industrie manufacturière ou les services, on passe à une qualité urbaine informée par le touristique qui tend à modifier le quotidien pour gommer les différences entre l'habitant-permanent et l'habitant-temporaire. L'urbanisation et la touristification constituent depuis plus d'un siècle deux processus/produits majeurs du développement des sociétés, entretenant toutes deux des relations à la fois d'interdépendance et de tension. Elles s'informent réciproquement, au sens où l'urbain est fondamentalement à l'œuvre dans le touristique et que ce dernier fait émerger de nouvelles urbanités. Au final, habitantstemporaires et habitants-permanents chercheraient tous, dans cette ville festive, à la fois les traits quotidiens d'un urbain "situé » et ceux extraordinaires d'un lieu touristique.

12 Cette réflexion prend donc l'aspect d'un essai théorique. Celui-ci repose, méthodologiquement parlant, sur le croisement entre, d'une part, la mobilisation et la critique d'un corpus théorique et, d'autre part, l'observation "en situation" d'expériences qui engagent des individus (touristes et/ou habitants), des objets (matériels, immatériels, symboliques), des environnements (contextes par lesquels et où se réalisent ces expériences). Il s'agit autant d'une posture scientifique que d'une hypothèse méthodologique. En milieu urbain, ce sont les paysages, les objets, les relations, les expériences, observées qui rendraient compte de la société, de ses évolutions. Les usages, désirs, besoins seraient ainsi auscultés à l'aune de leur concrétisation matérielle et ou expérientielle.

\section{Sur quelques expériences d'un « ailleurs sur place »}

13 Nantes et Lyon sont ici les terrains d'observation privilégiés de cet habiter touristiquement, en tant que villes représentatives de cette tendance touchant les grandes métropoles françaises, européennes, mondiales. Tout d'abord, les deux métropoles sont engagées dans une politique de renouvellement d'image et mettent en place un marketing urbain fort (Adam, 2016). Ensuite, les deux villes occupent régulièrement les premières places dans de nombreux classements vantant l'attractivité, l'art de vivre, la douceur, la richesse culturelle, etc. ${ }^{3}$. Enfin, toutes deux ont fait de l' «évènementialisation » des temps de l'urbain, et de l'urbain lui-même, 
l'alpha et l'oméga de l'accroissement de leurs visibilité, de leur renommée et de leur rayonnement (Gravari-Barbas, 2013).

14 De son côté, Nantes jouit d'un imaginaire lié au voyage, à l'exotisme, à l'aventure, grâce en partie à la littérature de Jules Verne, né à Nantes. À partir des années 1990, une politique urbaine visant à accroître l'attractivité et la qualité urbaines en lui donnant une nouvelle image est lancée. Cela va se traduire, notamment, par l'organisation de plusieurs évènements, tels que des festivals de musique du monde, classique, électronique, de cinéma, de photographie, de science-fiction, ainsi qu'une biennale d'art contemporain. Parallèlement, le projet de réaménagement et de renouvèlement urbain de l'île de Nantes est décidé. Le premier élément de ce projet est «le Grand Éléphant ", animal mécanique de bois et d'acier de 12 mètres de haut, qui promène 50 visiteurs par voyage sur cette île en mutation. Puis, en 2006, le " Hangar à bananes » prend ses quartiers. Anciens hangars du port maritime de Nantes-Saint-Nazaire, à proximité des Machines de l'île, ces quais réhabilités accueillent restaurants, bars, cafés-concerts, discothèques et lieux d'exposition. L'intervention architecturale est volontairement faible pour préserver le caractère emblématique des bâtiments. En 2007, l'artiste Daniel Buren intervient le long du Hangar à bananes et y place des anneaux en référence à la traite négrière. Cette œuvre est désormais l'un des symboles de la ville. Enfin, s'en suivra l'installation d'un «Carrousel des mondes marins " sur trois niveaux, en référence évidente à Jules Verne. Bref, tous les éléments sont mis en place pour proposer aux habitants permanents comme aux temporaires

une urbanité créée de toutes pièces, où le citadin-client peut trouver convivialité et ambiance festive et s'adonner à la consommation du plaisir (consommation à proprement parler, mais aussi loisirs, distractions ou activités préférées), tout en prenant un bain de foule et en se laissant agréablement surprendre par une ambiance de fête bon enfant. (Gravari-Barbas, 2006, p. 48-49)

Dans cette production d'une potentialité d'expériences d'un ailleurs sur place, un événement plus récent revêt un caractère singulier et illustre le propos de cette réflexion : le Voyage à Nantes (VAN). Depuis sa première édition en juin $2012^{4}$, chaque été, en suivant sagement une ligne verte de plus de 8 kilomètres de long peinte au sol, le VAN propose, en une sorte d'aventure citadine sous le régime du consentement libre et spontané, l'évasion, l'imprévu, l'éclectisme, la curiosité, etc. Ici, il est question d'adhérer à une exploration de lieux inconnus et/ou emblématiques, dans lesquels des œuvres offrent un regard nouveau sur la ville. Dans ce mode narratif implacable, il n'est pas rare d'observer que

les gens se marchent sur les pieds, se trouvent devant et dans des monceaux d'objets, entrecroisent jusqu'à ne plus les reconnaître les fils de leurs activités, embrouillent leurs situations de façon à engendrer des situations imprévues. (Lefebvre, 1970, p. 57)

16 Toutefois, en faisant le VAN, on ne fait pas que se marcher dessus, se cogner les uns aux autres, voire même entrer dans un magasin après qu'un habile commerçant a détourné la ligne verte pour la faire passer par son commerce. Le VAN, est un conte, un « il était une fois ", une ville renversée par l'art, offrant au touriste l'expérience d'une ville et d'une vie hors des sentiers battus, embarquant le résident dans l'avant-garde urbaine par un savant mélange de tradition, de multiculturalisme, d'imprévus, etc.

17 L'exploration se poursuit à présent avec l'exemple lyonnais, et plus particulièrement celui du quartier de Confluences ${ }^{5}$. Il s'agit ici d'apporter des éléments pour étayer la thèse que nous avançons, à savoir que tout acte de projetation, qu'il soit urbanistique 
et/ou architectural, a pour objectif de produire les conditions matérielles, immatérielles et symboliques d'un rapport au monde fondé sur l'entertainment. L'aménagement de ce quartier, débuté en 2003, donne aujourd'hui à voir un panel de réalisations produites par des architectes de renom: Christian de Portzamparc a dessiné l'Hôtel de région, Jakob + MacFarlane le Cube orange, Coop Himmelb(l)au a signé le controversé musée des Confluences. Ce quartier, nouveau et durable, vitrine métropolitaine, emprunte à l'exposition universelle une vision close, sorte de rétrospective voulant signifier une totale maitrise spatiale et temporelle en une quintessence de l'architecture et de l'urbanisme contemporains. Cette profusion, couplée à cet effet de miniaturisation, plonge habitants temporaires et permanents dans un état où distinguer la fantaisie de la réalité est délicat. Confluences illustre ainsi le fait que tout espace urbain, dans le cadre d'un marketing urbain féroce, serait une image donnée à voir dans l'espace public.

18 Comme la création architecturale se concentre sur la façade (Ibelings, 2003), les projets phares des villes sont avant tout destinés à être visités, observés et photographiés puis diffusés, à l'échelle la plus large possible, sur papier glacé (Jameson, 2007). Un nouvel urbanisme, dans lequel l'image et le récit triomphent (Matthey et Walther, 2007), s'instaure suivant une logique étrangement proche de celle des parcs à thème (Berdet, 2013). Architectes et urbanistes deviennent des sortes de metteurs en scène de tendances, et les productions urbaines et architecturales semblent n'être que des " panneaux publicitaires en forme de musées, de buildings (...) quand ce n'est pas tout un quartier transformé en Disneyland» (Garnier, 2011, p. 161) qui trône dans l'espace urbain. Confluences illustre que le caractère démonstratif d'un projet, le nom de son concepteur ou l'écho médiatique dont il bénéficie devient prioritaire sur ses fonctionnalités ou ses qualités d'usage. Le paysage urbain de Confluences, avec son musée, ou plus largement celui de Lyon, avec sa nouvelle tour Incity signée Valode et Pistre - la troisième tour la plus haute de France après la tour First à la Défense et la tour Montparnasse à Paris, surnommée « la gomme » en réponse à la tour Part-Dieu appelée «crayon » par les Lyonnais - offrent ainsi un raccourci dramatique du monde promis et tant attendu de la consommation totale. Sous leur double forme d'événement-projet (l'engagement dans une dynamique urbaine) et d'évènementconstruit (le symbole de cette dynamique dans le paysage urbain), ces productions sont la promesse d'une attractivité renouvelée (Adam et Laffont, 2018).

Ce que les villes de Nantes, en termes d'expériences urbaines, et de Lyon, en tant qu'environnement d'expression de cette ville festive, illustrent toutes deux, c'est le fait que le tourisme, opérateur et produit de la mondialisation, doit être intégré dans les logiques sociétales qui l'englobent et avec lesquelles il est toujours en interaction. L'urbanisation généralisée du monde fait converger objets, pratiques, valeurs, normes, qui appartiennent de plus en plus à une commune humanité. Mieux, celle-ci, en même temps qu'elle produit ses standards, fabrique la diversité dont elle a besoin pour fonctionner, y compris en valorisant des éléments de tradition. Car la « festivalisation de la ville " peut conduire à la vacuité et à la banalisation des espaces urbains et des villes elles-mêmes lorsque récits, aménagements et évènements semblent pauvres de sens, comme simplifiés à l'extrême, renvoyant trop fortement à la banalité et à l'imitation. Pour porter ses fruits, mais aussi pour dépasser les conflits entre habitants temporaires (touristes) et habitants permanents (résidents), l'enjeu est de parvenir à inscrire l'imaginaire au cœur de l'ordinaire. En cela, l'habitant résident et l'habitant temporaire deviennent tous deux bénéficiaires de cette mise en tourisme. Pour le 
premier, il s'agit d'avoir la sensation de n'être plus sous le régime du quotidien, dans la mesure où celui-ci est perpétuellement réinventé. Pour le second, il s'agit de gagner en authenticité dans la pratique de la ville désirée, dans la mesure où ce qui lui est offert n'est pas de l'ordre de l'imitation ou de l'adaptation de toute expérience qu'il pourrait vivre ailleurs.

20 Nantes et Lyon semblent partager une stratégie commune: promouvoir une image complexe de la ville et ne pas privilégier un "produit» touristique constitué uniquement de quelques lieux précis. À ce stade, les premières observations et analyses ne permettent pas de décrire avec finesse comment cela s'opère à Lyon, même s'il est possible d'avancer que cette stratégie se concrétise à l'échelle de la ville toute entière par l'intermédiaire de quartiers très spécialisés mais offrant une gamme importante et variée d'expériences urbaines ${ }^{6}$. S'agissant de Nantes, en revanche, il est possible de voir dans le VAN l'acteur du développement de cette stratégie. En effet, le Voyage à Nantes associe non pas des sites remarquables isolés mais met en parcours des ambiances variées, habitées et non pas muséifiées. Il n'est alors plus question de produire une ville exclusive, dédiée à l'un (le touriste) ou à l'autre (le résident), mais celle de l'un et de l'autre. Ainsi, la logique de l'événementiel fait dorénavant partie intégrante des politiques urbaines, et ce bien au-delà de l'occasionnel et de l'exceptionnel, pour devenir une dimension fondamentale de l'urbanité contemporaine.

Le Voyage à Nantes et Confluences-Lyon prônent une totale maîtrise de l'espace, du temps, des autres et de soi en une contradictoire expression miniature de la ville, qui en fait autant de maquettes d'exposition de cette nouvelle ère urbaine généralisée en marche. Que ce soit à Nantes ou à Lyon, ou encore ailleurs, le recours à l'événementiel, l'architecture-objet, la mise en récit des projets urbains sollicitent l'éprouvé émotionnel tant des résidents que des visiteurs. Ainsi, tout un arsenal de dispositifs sensibles, dont ceux observés ici, sert l'attractivité économique, résidentielle, touristique des métropoles. Qu'il s'agisse d'opérations d'envergure ou d'autres, plus ponctuelles et modestes, toutes sont censées satisfaire les envies et désirs des habitants, qu'ils soient permanents ou temporaires.

Comme cela a été partiellement illustré, l'objectif d'un événement comme le VAN et d'un projet urbain comme Confluences est non seulement d'attirer des consommateurs ponctuels que l'on pourrait, par simplification, qualifier de touristes, de répondre aux attentes des habitants actuels en termes d'urbanité, mais aussi de capter les futurs résidents. Ces derniers, mis en mouvement par un rapport esthétisant au quotidien, choisiraient telle ou telle ville pour des questions d'ambiance, de manifestions culturelles, de cadre de vie, de paysage urbain. Ainsi, ils seraient une sorte d'« avantgarde ", annonçant la migration des entreprises à forte valeur ajoutée. Rien de bien étonnant dès lors que l'urbanité ou encore le cadre de vie soient des critères privilégiés en matière de choix résidentiel dans les classements ou les politiques de marketing urbain.

Ces relations entre habiter touristiquement et urbanité, ici éclairées par les exemples nantais et lyonnais, mettent en question la séparation tranchée entre touristes et nontouristes, entre pratiques touristiques et vie quotidienne. Alors que les touristes cherchent à vivre comme des locaux, ces derniers consomment la ville dans laquelle ils habitent comme s'ils étaient des touristes. Une transgression réciproque se met alors en place qui, en abolissant les différences entre touristes et résidents, ainsi qu'entre 
espaces touristiques et espaces non touristiques, contribue à une recomposition urbaine et sociale.

\section{Habiter touristiquement : le droit à « quelle » ville?}

24 manifeste dans l'organisation des villes (concentrations, polarités, centralités, couplage
entre diversité et densité des réalités sociétales) et du rapport à l'urbain tel qu'il s'exprime aux travers de ces différentes expériences d'un ailleurs sur place. Le tourisme urbain est producteur et utilisateur d'urbanité, mais il peut également engendrer sa destruction, comme par exemple dans les processus de patrimonialisation (Duhamel et Knafou, 2007), dans lesquels les fonctions urbaines classiques sont temporairement ou définitivement sacrifiées au profit de leur mise en tourisme.

'effet d'une transformation à la fois matérielle et symbolique de l'espace urbain, par laquelle il s'agit de mettre en scène, à des dates et en des lieux fixés, les modalités de réappropriation ludique et conviviale des espaces publics, deux choses peuvent se produire : d'une part, une mise entre parenthèses des clivages et des antagonismes qui traversent ordinairement l'urbain; d'autre part, la mise en place d'une ville impersonnelle et interchangeable. En effet, au travers de l'idéal-type d'une population urbaine fervente, engagée et liée, célébrée à l'envi dans les presses locale, régionale et nationale, se profile le citadin modèle. À grands renforts d'animations, manifestations et autres événements soigneusement planifiés, il n'est pas exagéré de se demander si, sous couvert de rendre la ville aux habitants le temps d'une quelconque réjouissance, les acteurs de la production de l'urbain ne veulent pas faire du citadin d'aujourd'hui un spectateur (Gravari-Barbas, 2006). Car les objets physiques qui définissent l'environnement ne sont pas seuls à constituer le lieu. Celles et ceux qui occupent l'espace sont des acteurs qui donnent la couleur locale et l'ambiance du lieu. Érigée en marque déposée sous divers labels changeants, toute ville festive touristiquement habitée en appelle à la coopération massive et dynamique de figurants sur les espaces publics scénographiés. Seul cela, en donnant corps à la nouvelle identité recherchée, servira à une ville pour se démarquer de ses rivales. Autrement dit, aujourd'hui, dans la civilisation ludico-commerciale, les «consom'acteurs» (Gombault, 2011) sont non seulement les cibles mais surtout les vecteurs du marketing urbain. Habiter touristiquement exige que, constamment, les individus réagissent et interprètent la multitude de signes et d'images dont ils sont bombardés (Guiheux, 2017). La marchandisation qui façonne et assimile l'expérience permet ainsi à l'individu de modeler sa propre conscience grâce au choix. Ainsi, chacun se différencie des autres pour attester de ce qui lui reste de liberté et de singularité en optant pour les consommations conformes à ce qu'il estime devoir constituer et véhiculer sa propre image de marque.

L'exploitation symbolique de la création architecturale, urbanistique, culturelle, en tant que signe, savamment concoctée fournit non seulement le décorum et la matière pour la mise en œuvre de politiques urbaines censées rendre la ville festive, récréative mais qui en conditionnent de fait les modalités. En tant qu'illustrations des relations et tensions entre urbanité et tourisme, le Voyage à Nantes et Confluences sont pensés comme des espaces favorisant la mise en scène de la production de périmètres marchands organisés afin de «créer un ensemble attrayant, dense, animé, dans lequel

Mondes du Tourisme, 15 | 2019 
le parcours du visiteur est censé s'inscrire dans une expérience globale lui apportant des sensations inédites » (Gravari-Barbas, 2006, p. 51).

En concentrant une majeure partie des investissements dans ces opérations phares, souvent au détriment du financement d'activités associatives, culturelles, sociales, toutes locales, les acteurs de la production urbaine misent sur une gratification individuelle et façonnent l'urbain. Ces pratiques calibrent une certaine esthétique, un certain urbanisme, une certaine architecture, tous trois sémiotisation de l'environnement produit. En conséquence, l'agenda évènementiel de villes comme Lyon et Nantes ne se concentre plus sur quelques moments et quelques sites clés. Tracté par ces locomotives, c'est l'espace urbain dans son ensemble qui se met perpétuellement en scène. En outre, cette isochronie doublée d'une isotopie est censée galvaniser les populations locales et de passage pour produite une identité collective sur fond de récit de territoire quelque peu alternatif; comme à Nantes, où la belle endormie, industrieuse devient une ville moderne, dynamique, culturelle, à mi-chemin entre Amsterdam et Berlin.

28 La démocratie de marché, prise au sens de projet civilisateur universel, semble accoucher d'un homme nouveau, le « citoyen global », dont la production en série va de pair avec la reproduction à l'identique et sans heurts d'une société de citadins disciplinés. Les tensions qui ne pourront être maîtrisées seront dérivées vers des simulacres déréalisant l'existant pour donner à la production de l'urbain un visage d'autant plus avenant qu'il sera convivial et festif. La population sera postulée homogène, comme celle du monde globalisé. Seules seront admises, et même recommandées, comme différences des tranches d'altérité programmées que l'on pourra s'offrir sous forme de spectacles culturels, exotiques ou revivalistes, à partir de l'exploitation de patrimoines locaux dûment estampillés.

Cela était avancé précédemment: nous sommes entrés dans l'ère d'un service surmesure... de masse. Et, dans cette ère, "sous-produit de la circulation des marchandises, la circulation humaine considérée comme une consommation, le tourisme, se ramène fondamentalement au loisir d'aller voir ce qui est devenu banal » (Debord, 1967, p. 164). Ainsi, l'urbain contemporain est à vendre ou à jouer, parfois même les deux (Page et Hall, 2003), prenant la « forme d'un parc à thème qui exfiltre les scories de la réalité vraie» (Bégout, 2012, p. 165). L'urbanité, elle, oscille entre le mercantile et le convivial. Quant aux pratiques qui fondent ce nouveau - et en passe de devenir unique - mode d'habiter, elles peuvent devenir une somme veine d'ajustages dont le seul but - en aucun cas le sens - est de combler l'instant d'une manière aseptisée.

30 Henri Lefebvre posait comme fondamental le droit à l'accès à la vie urbaine, aux lieux de rencontres, d'échanges et de rassemblements. Plus que jamais, la thématique de l'appropriation de l'espace, a fortiori celle de l'espace public, devrait être investiguée et, par conséquent, politisée dans le contexte de la production contemporaine de l'urbain, plus particulièrement lorsqu'on se focalise sur les rapports entre urbanité et habiter touristiquement. Ce droit à la ville «ne se réduit donc pas à un droit d'accès individuel aux ressources incarnées par la ville: c'est un droit à nous changer nous-même en changeant la ville de façon à la rendre la plus conforme à notre désir le plus cher " (Harvey, 2011, p. 8). Il y a peu de chances que ce désir ne se résume qu'à un vaste apprêtage et essayage permanent d'urbanité divertissante. 
Que ce soit dans le cas du Voyage à Nantes ou du quartier Confluences à Lyon, on assiste à la production de lieux autosuffisants, d'espaces épurés et fermés sur euxmêmes, dans lesquels peuvent s'exprimer des désirs et des passions normalisables et pouvant être considérés comme normalisés. Ces deux exemples illustrent cette même ambition, qui est de coloniser tout le tissu urbain par une série d'opérations, de manifestations, de performances, d'évènements fondés sur le socle du divertissement et nous enjoignant à vivre des expériences urbaines combinant plusieurs aspirations touristiques (jeu, consommation, découverte, culture, rencontres, etc.). À l'époque de l' entertainment généralisé, la distinction entre habiter touristiquement et habiter n'a plus cours. À la faveur de différents processus d'appropriation et de dépossession (patrimonialisation, festivalisation, ségrégation, gentrification), l'hybridation entre résidents et touristes serait en train de produire l'homo touristicus et de l'étendre à l'ensemble du monde urbain contemporain. Les publics non cibles deviennent des spectateurs, contemplant la réussite des uns, apprenant patiemment les différents codes qui leur permettraient, à eux aussi, en quelque sorte, «d'en être ». Tout comme le crédit, qui non seulement permet l'acquisition d'une maison individuelle mais aussi d'accéder au statut de membre à part entière de la société (Mercier, 2006), faire un tour d'Éléphant à Nantes ou acheter différents marqueurs sociaux au centre commercial de Confluences devient une manifestation de son adhésion à la société, et à l'urbanité qui va avec.

Désormais, afin de consolider ces interprétations, l'orientation prise par cette recherche initiée à Nantes et à Lyon est double. Tout d'abord, elle consiste à saisir et analyser les perceptions et appropriations d'espaces «mis en tourisme» par les habitants et les touristes. Puis, de manière concomitante, il est question d'analyser, et donc de comprendre, le sens conféré par les acteurs de la ville (architectes, urbanistes, paysagistes, politiques, designers, etc.) à ces mêmes espaces, tant dans leurs aspects matériels qu'immatériels.

Dans un spectaculaire renversement qui prend le contre-pied de la thèse d'Hannah Arendt publiée en 1961, culture et loisirs deviennent compatibles, car c'est bien le politique et l'économique, notamment par l'architecture et l'urbanisme, qui, souhaitant constituer un monde commun, institutionnalisent et orchestrent la monstration collective de la satisfaction d'un besoin vital, individuel et égoïste par l'organisation d'évènements institués. Cela ouvre une piste à investiguer, à savoir si «l'urbain conçu et vécu comme pratique sociale est en voie de détérioration et peut-être de disparition » (Lefebvre, 1989, p. 14). 


\section{BIBLIOGRAPHIE}

Matthieu ADAM et Georges-Henry LAFFONT, « Conjuguer singularité et conformité pour se positionner sur le marché international de l'urbain. Confluence et le renouvellement de l'image de Lyon », Confins, n 36, 2018 [http://journals.openedition.org/confins/14614 ; DOI : 10.4000/ confins.14614].

Tommy D. ANDERSSON, « The Tourist in the Experience Economy », Scandinavian Journal of Hospitality and Tourism, vol. 7, n 1, 2007.

Bruce BÉGOUT, Suburbia, Inculte, 2012.

Marc BERDET, Fantasmagories du capital. L'invention de la ville marchandise, La Découverte, 2013.

Guy DEBORD, La société du spectacle, Champ libre, 1967.

Équipe MIT, Tourismes 1. Lieux communs, Belin, 2002.

Philippe DUHAMEL, « La troisième révolution touristique - Évolution des lieux et des territoires », Mondes du Tourisme, hors-série, 2016 [http://journals.openedition.org/tourisme/1263].

Philippe DUHAMEL et Rémi KNAFOU, Les mondes urbains du tourisme, Belin, 2007.

Jean-Pierre GARNIER, « Ne pas se tromper de cible », Espaces et sociétés, n 146, 2011.

Maria GRAVARI-BARBAS, Aménager la ville par la culture et le tourisme, éditions du Moniteur, 2013.

Maria GRAVARI-BARBAS, La ville à l'ère de la globalisation des loisirs, éditions Espaces, 2006.

Maria GRAVARI-BARBAS, La ville festive. Espaces, expressions, acteurs, habilitation à diriger des recherches, Université d'Angers, 2000.

Maria GRAVARI-BARBAS et Édith FAGNONI, « Introduction », dans Maria GRAVARI-BARBAS et Édith FAGNONI (dir), Tourisme et métropolisation. Comment le tourisme redessine Paris, Belin, coll.

« Mappemonde », 2013.

Anne GombaUlT, « Tourisme et création : les hypermodernes ", Mondes du tourisme, $\mathrm{n}^{\circ}$ 4, 2011

[http://journals.openedition.org/tourisme/449 ; DOI : 10.4000/tourisme.449].

David HARVEY, Le capitalisme contre le droit à la ville. Néolibéralisation, urbanisation, résistances, éditions Amsterdam, 2011.

André-Frédéric HOYAUX, « Habiter : se placer plaçant et se penser pensant », Annales de géographie, $n^{\circ} 704,2015$.

Fredric JAMESON, Le postmodernisme ou la logique culturelle du capitalisme tardif, ENSBA Paris, 2007.

Hans IBELINGS, Supermodernisme : l'architecture à l'ère de la globalisation, Hazan, 2003.

Rémi KNAFOU, «L'urbain et le tourisme : une construction laborieuse », dans Philippe DUHAMEL et Rémi KNAFOU (dir), Les mondes urbains du tourisme, Belin, 2007.

Georges-Henry LAFFONT et Lionel PRIGENT, « Paris transformé en décor urbain », Téoros, n 30-1, 2011.

Scott LASH et John URRY, Economy of signs and space, Sage London,1994.

Henri LEFEBVRE, La révolution urbaine, Gallimard, 1970. 
Henri LEFEBVRE, La production de l'urbain, Anthropos, 2005 (1974).

Henri LEFEBVRE, «Quand la ville se perd dans la métamorphose planétaire », Le Monde Diplomatique, 1989.

Michel Lussault, « Urbanité », dans Jacques LÉVy et Michel lussault (dir), Dictionnaire de la géographie et de l'espace des sociétés, Belin, 2003.

Michel Lussault et Mathis sтоск, « Tourisme et urbanité ", dans Philippe duHAmEl et Rémi KNAFou (dir.), Les mondes urbains du tourisme, Belin, 2007.

Robert MAITLAND, « Everyday Life as a Creative Experience in Cities ", International Journal of Culture, Tourism and Hospitality, vol. 4, $\mathrm{n}^{\circ}$ 3, 2010.

Laurent MATTHEY et Olivier WALTHER, « Avant-gardes et élites : agents de reproduction ou de transformation de la société ? Une présentation ", Articulo - Journal of Urban Research, n 3, 2007.

GuY MERCIER, « La norme pavillonnaire : mythologie contemporaine, idéal urbain, pacte social, ordre industriel, moralité capitaliste et idéalisme démocratique ", Cahiers de géographie du Québec, $n^{\circ} 50,2006$

Marie-José MONDZAIN, L’image naturelle, Le nouveau commerce, 1995.

Stéphane NAHRATH et Mathis STOCK, «Urbanité et tourisme : une relation à repenser ", Espace et sociétés, $\mathrm{n}^{\circ} 151,2012$.

Stephen J. PAGE et Colin Michael HALL, Managing urban tourism, Pearson Education, 2003.

Greg RICHARDS, «Creativity and Tourism: The State of the Art », Annals of Tourism Research, vol. 38, $n^{\circ} 4,2011$.

Hélène SALLET-LAVOREL, Pour un tourisme participatif en Île-de-France. Encourager le rapprochement entre touristes et visiteurs, rapport présenté à l'IAURIF, 2003.

Jean-Louis SCHAEFFER, L'expérience esthétique, coll. « NRF Essais », Gallimard, 2015.

Mathis STOCK, « European cities: Towards a recreational turn? ", HAGAR Studies in Culture, Polities and Identities, vol. 7, $\mathrm{n}^{\circ}$ 1, 2007.

Yi-Fu TUAN, Espace et lieu : la perspective de l'expérience, InFolio, 2006 (1977).

Jean-Didier URBAIN, Secrets de voyage. Menteurs, imposteurs et autres voyageurs invisibles, coll. « Essais », Payot, 1998.

John URRY, The tourist gaze: Leisure and Travel in Contemporary Societies, Sage, 1990.

\section{NOTES}

1. La méthode, tant dans son positionnement que dans ses modalités, sera explicitée dans l'article.

2. La sérendipité exprime le rôle du hasard dans les découvertes, grâce auquel on trouve quelque chose que l'on ne cherche pas.

3. Une recherche sur Internet par association de mots clefs telle que "ville/qualité/Nantes", «classement/ville/Lyon", la consultation des sites institutionnels des deux villes ou d'organismes dits indépendants, la publication de numéros spéciaux dans la presse, etc. illustrent ce constat. 
4. Cet événement reprend le principe de la biennale d'art contemporain « l'Estuaire » $(2007,2009$, 2011) et installe de manière éphémère ou ponctuelle des créations d'art contemporain en un «musée à ciel ouvert ». L'originalité des œuvres et de leur intégration dans le paysage urbain renvoient à l'idée d'une ville, Nantes, qui est là où on ne l'attend pas.

5. Confluences est un vaste projet urbain de requalification de friches industrielles à la confluence du Rhône et de la Saône. Sur 170 hectares, se répartissent programme de logement public (1 800 unités), programme privé ( $240000 \mathrm{~m}^{2}$ de bureaux et de commerces) et équipements publics visant différentes échelles de populations et de territoires, dont le désormais célèbre musée des Confluences à l'architecture et au coût de construction discutés.

6. Les quartiers lyonnais comme la Presqu'île, le Vieux-Lyon et la colline de Fourvière, la CroixRousse, Guillotière ou encore la Part-Dieu, attracteurs touristiques et résidentiels, ont des différences très marquées mais n'en sont pas pour autant isolés. En effet, le Rhône et la Saône, par les récents aménagements de leurs quais, fonctionnent comme des traits d'union et permettent une découverte et une appréciation globale de la ville par incrémentation.

\section{RÉSUMÉS}

En s'inscrivant dans la continuité des travaux sur les liens entre tourisme et production de l'espace, cette contribution défend la thèse que nous sommes entrés dans une nouvelle ère dépassant le recreational turn. Il n'y aurait plus, à présent, de simples relations d'interdépendance et des tensions entre urbanisation et touristification: l'homme habiterait en touriste. Tout espace urbain serait apprêté par et pour le tourisme ; tout acte de projetation urbanistique et/ou architecturale viserait à produire les conditions d'un rapport au monde fondé sur l'entertainment. Pour défendre cette thèse, une double étude du Voyage à Nantes et de Confluences-Lyon, comme illustrations emblématiques de cette nouvelle ère, et des travaux portant sur les spatialités contemporaines où urbanité, tourisme, production de l'urbain et habiter sont intriqués seront mobilisés.

Continuing work on the links between tourism and spatiality, this text supports the thesis that we have entered a new era beyond the "recreational turn". Now there would no longer be mere interdependent relations and tensions between urbanisation and tourism: man would inhabit the world as a tourist. Any urban space would be designed by and for tourism; any act of urban and/ or architectural planning would aim to produce the conditions for a relationship with the world based on entertainment. To defend this thesis, a double study of the Voyage à Nantes and Confluences-Lyon, as emblematic illustrations of this new era, and of literature on contemporary spatialities, where urbanity, tourism, urban production and inhabiting are entangled, will be mobilised.

\section{INDEX}

Mots-clés : habiter, urbanité, tourisme, production de l'espace, expérience, Voyage à Nantes, Lyon-Confluences

Keywords : inhabiting, urbanity, tourism, spatiality, experience, Voyage à Nantes, LyonConfluences 


\section{AUTEUR}

\section{GEORGES-HENRY LAFFONT}

Docteur en aménagement de l'espace et urbanisme

Maître de conférences à l'ENSA Saint-Étienne, champ "Ville et territoire »

Chercheur permanent à EVS - Isthme, UMR 5600 et labEX IMU, Université de Lyon

Chercheur associé au laboratoire CITERES, UMR 7324 de l'Université de Tours - Urbanisme, aménagement de l'espace et géographie

Courriel : georges-henry.laffont@st-etienne.archi.fr ; georges-henry.laffont@univ-tours.fr 